externally. They were then placed in $10 \%$ formalin for four to five minutes. Thereafter they were removed one by one to a tube of sterile broth and shaken up. From this tube they were transferred to a second broth tube and shaken. Finally from this second tube each pupa was removed to an agar slope and mashed up with a strong platinum loop. The two broth tubes and the series of agar slopes were then incubated at $37^{\circ} \mathrm{C}$. Next day both the broth tubes were sterile but all the agar slopes showed abundant growth in which B. pyocyaneus was present. After 48 hours the broth tubes were still sterile but on the third day one broth tube (the second) showed growth. This slight discrepancy, however, in no way invalidates the general conclusion arrived at by $\mathrm{Mr}$ Bacot that the pupal interior may become heavily infected by organisms taken up during the larval stage. By further improvements in technique it may be possible to eliminate entirely any growth-effects before the final mashing-up process ${ }^{1}$.

\title{
EDTTORIAL NOTE.
}

Dr Graham-Smith, who has been carrying out investigations for the Local Government Board, has obtained some very interesting results.

Blow-fly (Calliphora erythrocephala) maggots were fed on meat infected with the spores of $B$. anthracis. When full fed the maggots were transferred to clean cages in which they pupated. The flies emerged about three weeks after the larvae had fed on the infected meat. Cultures were made from the organs of 51 of those flies, in some cases shortly after emerging, and in some cases when the flies were a few days old. B. anthracis was obtained from 26 flies. B. anthracis was also obtained from agar plates on which the flies had walked, from faeces deposited by them, and from syrup on which they were allowed to feed. Several of the cultures obtained were proved to be virulent.

These investigations will shortly be published in Reports to the Local Government Board on Public Health and Medical Subjects.

G. H. F. N.

\footnotetext{
1 Since this was written I have devised a simple but efficient method of examining the bacterial content of the pupal interior in $M$. domestica. The pupa is held lightly between the left thumb and forefinger so that its blunt extremity is free. With a small searingiron this extremity is seared and at the same time flattened. It is then pierced by a fine capillary pipette controlled by a rubber teat. The pupal contents are stirred up by the extremity of the pipette and finally drawn up into the tube whence they are immediately squirted on to plates. The whole process takes less than a minute to perform.

Recently I have been successful in isolating $B$. typhosus from pupae the larvae of which have fed on this organism. The experiments will be published shortly.
} 